\title{
Publisher Correction: Bridging homogeneous and heterogeneous catalysis by heterogeneous single-metal-site catalysts
}

Xinjiang Cui (D), Wu Li, Pavel Ryabchuk, Kathrin Junge and Matthias Beller (D

Correction to: Nature Catalysis https://doi.org/10.1038/s41929-018-0090-9, published online 12 June 2018.

In the version of this Review originally published, the titles of Table 1 and Table 3 were mistakenly swapped. The title for Table 1 should be "Single Pt site catalysed selective hydrosilylation of various olefins", and the title for Table 3 should be "Synthesis and applications of single-metal-site catalysts". These errors have now been amended.

Published online: 11 February 2019

https://doi.org/10.1038/s41929-019-0248-0 